\title{
Desenlace de los pacientes pediátricos con falla medular tratados en un centro de alta complejidad
}

\author{
Outcomes in pediatrics patients diagnosed with bone marrow failure disorders \\ treated in a tertiary care center
}

\author{
Diego Medina Valencia ${ }^{\mathrm{a}, \mathrm{b}}$, Mayra Estacio ${ }^{\mathrm{c}}$, Ana Clarete ${ }^{\mathrm{b}}$, Sofía Timarán ${ }^{\mathrm{b}}$, \\ Eliana Manzi ${ }^{\mathrm{b}, \mathrm{c}}$, Estefanía Beltrán Gómez ${ }^{\mathrm{c}}$, Alexis A. Franco ${ }^{\mathrm{a}, \mathrm{b}}$
}

\author{
aFundación Valle del Lili, Servicio de Hemato-Oncología Pediátrica y Trasplante de Médula Ósea. Cali, Colombia. \\ bFacultad de Ciencias de la Salud, Universidad Icesi. Cali, Colombia. \\ 'Fundación Valle del Lili, Centro de Investigaciones Clínicas. Cali, Colombia.
}

Recibido: 30 de diciembre de 2019; Aceptado: 17 de mayo de 2020

\section{¿Qué se sabe del tema que trata este estudio?}

Los síndromes de falla medular, tienen baja prevalencia. Predomina la etiología adquirida. En el síndrome de falla medular adquirido el tratamiento de primera línea es trasplante alogénico de familiar idéntico y si no tiene esta posibilidad terapia de inmunosupresión.
¿Qué aporta este estudio a lo ya conocido?

Las tasas de respuesta a terapia de inmunosupresión son inferiores a las reportadas en países desarrollados. En el síndrome de falla medular adquirido, el trasplante alogénico incluyendo haploidéntico es una buena opción como terapia de rescate.

\section{Resumen}

Los síndromes de falla medular (SFM) son trastornos infrecuentes, con una incidencia anual de 2-4 casos por millón. Las opciones de tratamiento incluyen terapia de inmunosupresión (TIS) y restauración de la hematopoyesis con trasplante de progenitores hematopoyéticas (TPH). Objetivo: Analizar los desenlaces de pacientes pediátricos diagnosticados con SFM tratados en una institución de alta complejidad. Pacientes y Método: Estudio retrospectivo de pacientes pediátricos con diagnóstico de SFM que consultaron a la Fundación Valle del Lili, Cali. Se realizo análisis estadístico descriptivo según SFM adquirida (SFMA) y SFM congénita (SFMC). Los desenlaces incluyeron: tratamiento, complicaciones, supervivencia global (SG) en los trasplantados, calculada con el método KaplanMeier. Resultados: Se incluyeron 24 pacientes con SFM, edad 6,5 \pm 4 años, 50\% mujeres. El 58\% fueron SFMC, 9 con anemia de Fanconi, 2 disqueratosis congénita, 2 trombocitopenia amegacariocítica congénita, uno anemia Diamond-Blackfan. Doce pacientes con TPH tuvieron SG a 5 años de $83 \%$. SFMA correspondió al 42\%, 6 recibieron TIS-TPH, 3 TIS y 1 TPH, la SG del grupo con TIS-TPH fue $86 \%$. Seis pacientes fallecieron, $4 / 6$ relacionadas con infección. Conclusiones: En esta serie fue mayor
Palabras clave: Síndrome de Falla Medular;

Fanconi;

Pancitopenia;

Trasplante de

Células Madre;

Inmunosupresión;

Timoglobulina;

Aspirado;

Biopsia;

Transfusión

Correspondencia:

Diego Medina Valencia

diego.medina@fvl.org.co 
el número de casos con SFMC. La SG de los pacientes llevados a TPH es comparable con la reportada en estudios recientes. La causa de muerte predominante fue infecciosa que también se ha reportado previamente. El tratamiento instaurado en los pacientes de esta serie mostró resultados favorables en un centro de alta complejidad en un país latinoamericano.

\section{Keywords:}

Bone Marrow Failure Syndromes; Fanconi Anemia; Pancytopenia; Stem Cell Transplantation; Immunosuppression; Thymoglobulin; Biopsy; Transfusion

\section{Introducción}

El síndrome de falla medular (SFM) es un trastorno poco frecuente, con una incidencia anual estimada de 2-4 casos por millón; el rango etario de presentación más frecuente va desde los 10-25 años y después de los 60 años afectando por igual a ambos sexos ${ }^{1}$. Se caracteriza por la disminución o ausencia de precursores hematopoyéticos en la médula ósea, alterando su función hematopoyética ${ }^{2,3}$. Se presentan típicamente con citopenias persistentes en al menos un linaje celular, que pueden progresar a pancitopenia ${ }^{4}$.

Respecto a su etiología, se puede clasificar como síndrome de falla medular congénita (SFMC) y adquirida (SFMA) $)^{5,6}$. Los SFMA representan el 85-90\% de los casos, y su tratamiento primario es terapia inmunosupresora (TIS) o el trasplante de progenitores hematopoyéticos (TPH), dependiendo de la disponibilidad de donante adecuado ${ }^{6,7}$. En cuanto a los SFMC, estos corresponden al 15-20\% de los casos, en los cuales el tratamiento usualmente corresponde a TPH idéntico $y$, en algunos casos, haploidéntico ${ }^{8}$. Entre las principales etiologías de los SFMC en niños se encuentran la anemia de fanconi (AF), disqueratosis congénita (DC), síndrome de Shwachman-Diamond (SDS) y trombocitopenia amegacariocítica congénita (CAMT). Estos síndromes pueden presentarse con o sin anomalías físicas y no necesariamente demuestran pancitopenia completa, particularmente durante las primeras fases de la enfermedad ${ }^{3}$. El objetivo de este estudio es describir el desenlace de pacientes pediátricos tratados por síndrome de falla medular entre 2011-2017 en una institución de alta complejidad.

\section{Pacientes y Método}

\section{Población estudiada}

Estudio observacional, tipo serie de casos, de pacientes pediátricos con diagnóstico de SFM, que consultaron entre 2011-2017 a la Fundación Valle del Lili, institución de alta complejidad y centro de referencia en la ciudad de Cali, para pacientes que requieren TPH. Dentro de la estrategia de muestreo se utilizaron los códigos CIE-10 relacionados con SFM registrados en la historia clínica, obteniéndose un total de 2.491 pacientes con edades entre los 0 a 17 años que consultaron en el período del estudio. Posteriormente se aplicaron los siguientes criterios de selección: pacientes tratados en la Institución, con diagnóstico de SFM por síntomas clínicos, estudios paraclínicos y comprobado por biopsia de medula ósea. El diagnóstico de AF se realizó a través del Test de Fragilidad Cromosómica. El diagnóstico de DC se hizo con panel de secuenciación de nueva generación (NGS) de falla medular, CAMT con biopsia de medula ósea y anemia Diamond-Blackfan (ADB) según los criterios diagnósticos clásicos 9 . Se excluyó un paciente en quien no se logró determinar 
la etiología del SFM. Este estudio fue aprobado por el Comité de Ética Institucional.

\section{Tratamiento SFM}

Las opciones terapéuticas utilizadas se basaron en las guías de atención para pacientes con SFMA, dando un enfoque multidisciplinario, indicando como primera opción TPH si se contaba con donante idéntico o TIS en pacientes sin indicación de TPH o que no disponían de donante idéntico relacionado ${ }^{6}$. En el SFMC el tratamiento está basado en soporte transfusional, andrógenos y TPH en los casos severos o con mala respuesta ${ }^{10}$.

\section{Trasplante}

Regímenes de condicionamiento: El régimen de condicionamiento para cada paciente se realizó según el tipo de donante, basados en fludarabina ${ }^{11,12}$. En el caso de los trasplantes con donante haploidéntico se usó protocolo de intensidad reducida basado en el régimen de Baltimore ${ }^{13,14}$.

\section{Profilaxis de la enfermedad de injerto contra huésped (EICH)}

Los esquemas de profilaxis para EICH utilizados variaron según el tipo de trasplante realizado. Para los trasplantes de donante familiar idéntico se usaron regímenes basados en ciclosporina combinada con metotrexato ${ }^{15,16}$. En los trasplantes haploidénticos se usó el régimen basado en ciclofosfamida postrasplante ${ }^{17-19}$.

\section{Severidad de SFM}

La severidad del SFM se clasificó según los criterios de Camitta, como SFM severo si reúne al menos 2 de tres características: recuente absoluto de neutrófilos (RAN) $<0,5 \times 10^{9} / \mathrm{L}$, recuento de plaquetas $<20$ $\times 10^{9} / \mathrm{L}$ o recuento de reticulocitos $<20 \times 10^{9} / \mathrm{L}$, SFM muy severo con las mismas características de la SFM severa, pero con RAN $<0,2 \times 10^{9} / \mathrm{L}$, y SFM no severo cuando no cumple ninguna de las características de SFM severo o muy severo ${ }^{20}$.

\section{Criterios de respuesta al tratamiento con terapia inmunosupresora (TIS)}

Respuesta completa: lograr la independencia transfusional y recuentos de Hemoglobina $>11 \mathrm{~g} / \mathrm{dL}$ + plaquetas $>100 \times 10^{9} / \mathrm{L}+$ neutrófilos $>1,5 \times 10^{9} / \mathrm{L}$. Respuesta parcial: Tener independencia transfusional, pero sin lograr los valores de respuesta completa en el hemograma, confirmado en 2 controles con una diferencia de 4 semanas. No respuesta: no tener independencia transfusional ${ }^{6}$.

\section{Análisis estadístico}

Se hizo un análisis estadístico descriptivo para todas las variables consideradas, separando a los pa- cientes en dos grupos: SFMA y SFMC. Las variables categóricas se resumen en proporciones y las variables continuas se expresan como promedios \pm desviación estándar (DE) o mediana con su rango intercuartil (RIC), según la distribución de la variable. Los desenlaces primarios del estudio fueron: tratamiento, complicaciones, la supervivencia global (SG) en meses, calculada a partir de la fecha del diagnóstico o fecha del trasplante, hasta la fecha de fallecimiento o del último seguimiento en la institución, analizada con el método Kaplan-Meier, con el software estadístico STATA $^{\circledR} 12.1$.

\section{Resultados}

\section{Población estudiada}

Entre 2011-2017, 24 pacientes pediátricos fueron tratados por SFM en el servicio de hemato-oncología pediátrica. La figura 1 muestra el flujo de los pacientes en el estudio.

Las características demográficas y clínicas se describen en la tabla 1 y tabla 2 . La edad promedio fue de 6,5 \pm 4 años, el 50\% era de sexo femenino, y el síntoma más frecuente fue síndrome anémico $(87 \%)$. El diagnóstico más frecuente fue SFMA (42\%), seguida de AF (37\%). Hubo 19 (79\%) pacientes que fueron llevados a TPH y tuvieron una mediana de seguimiento de 22 (RIC: $13-65)$ meses y SG del $84 \%$ a 5 años.

\section{Síndrome de falla medular adquirida (SFMA)}

Diez pacientes $(42 \%)$ se incluyeron en este grupo. De éstos, 9/10 recibieron tratamiento con TIS (un caso extrainstitucional) de los cuales 6/9 fueron trasplantados por no respuesta al tratamiento. Hubo un paciente que fue llevado directamente a TPH con donante idéntico. La SG a 5 años en este grupo fue del $70 \%$; los pacientes trasplantados tuvieron una SG a 5 años de $86 \%$ vs $33 \%$ en los no trasplantados (figura 2). Tres pacientes fallecieron, uno por falla multiorgánica después de ser llevado a TPH y dos por infección después de ser tratados con globulina antitimocítica (ATG) sin TPH.

\section{Síndrome de falla medular congénita (SFMC)}

Hubo 14 (58\%) pacientes en este grupo, la mayoría 9/14 con AF. Doce pacientes recibieron TPH (dos extrainstitucionalmente con seguimiento posterior en nuestra institución) y dos pacientes recibieron solo soporte transfusional. La SG a 5 años fue de $83 \%$ en los pacientes trasplantados (figura 2). Tres pacientes fallecieron después del TPH, dos por causas asociadas al TPH: infección por adenovirus y citomegalovirus y una por compromiso pulmonar y hepático secundario a DC. 
Figura 1. Flujograma de pacientes con SFM. SFM: Síndrome de falla medular; SFMA: Síndrome de falla medular adquirida; SFMC: Síndrome de falla medular congénita; TPH: Trasplante de progenitores hematopoyéticos; ATG: Globulina antitimocítica.

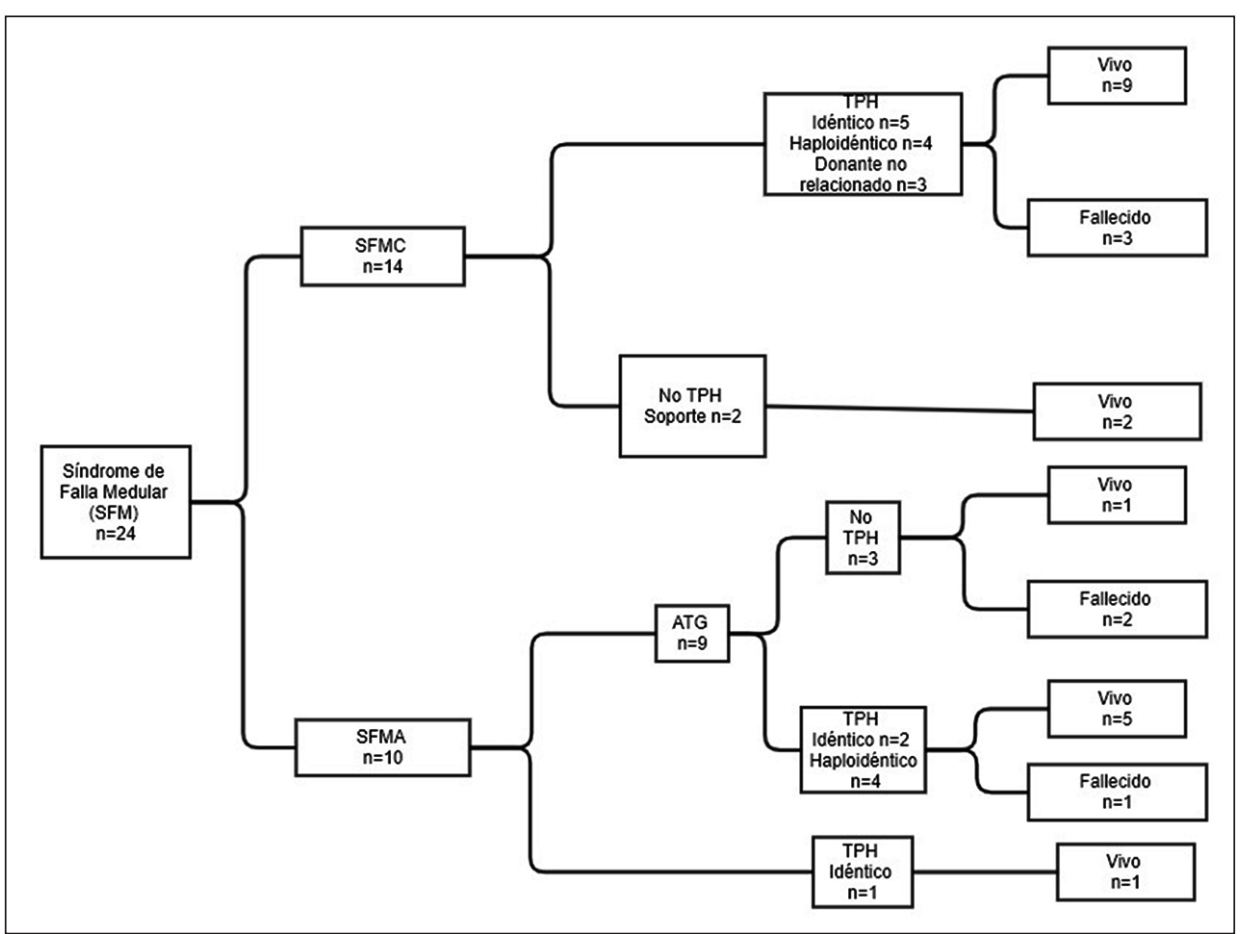

Tabla 1. Características demográficas y clínicas de pacientes pediátricos con SFM en un centro de alta complejidad

\begin{tabular}{|c|c|c|c|}
\hline Característica & Total $n=24$ & SFMA $n=10$ & SFMC $n=14$ \\
\hline \multicolumn{4}{|l|}{ Edad al diagnóstico, años } \\
\hline Promedio \pm DE & $6,5 \pm 4,4$ & $7,8 \pm 4,3$ & $5,5 \pm 4,3$ \\
\hline Rango & $0,03-15$ & $1-15$ & $0,03-12$ \\
\hline Sexo femenino, n (\%) & $12(50)$ & $4(40)$ & $8(57)$ \\
\hline \multicolumn{4}{|l|}{ Síntomas, n (\%) } \\
\hline Síndrome hemorrágico & $17(68)$ & $7(70)$ & $9(64)$ \\
\hline Síndrome anémico & $21(87)$ & $10(100)$ & $11(78)$ \\
\hline Infecciones graves y/o recurrentes & $9(37)$ & $5(50)$ & $4(29)$ \\
\hline \multicolumn{4}{|l|}{ Etiología del SFM, n (\%) } \\
\hline Aplasia medular adquirida & $10(42)$ & $10(100)$ & $0(0)$ \\
\hline Anemia de Fanconi (AF) & $9(37)$ & $0(0)$ & $9(64,3)$ \\
\hline Disqueratosis congénita (DC) & $2(8)$ & $0(0)$ & $2(14,3)$ \\
\hline Trombocitopenia amegacariocítica congénita (CAMT) & $2(8)$ & $0(0)$ & $2(14,3)$ \\
\hline Anemia Diamond Blackfan & $1(4)$ & $0(0)$ & $1(7,1)$ \\
\hline Frecuencia de SFM severa y muy severa, n (\%) & $14(70)$ & $10(100)$ & $7(50)$ \\
\hline ATG & $9(37)$ & $9(90)$ & - \\
\hline Trasplante, n (\%) & $19(79)$ & $7(70)$ & $12(86)$ \\
\hline Idéntico & $8 / 19$ & $3 / 7$ & $5 / 12$ \\
\hline Haploidéntico & $8 / 19$ & $4 / 7$ & $4 / 12$ \\
\hline Donante no relacionado & $3 / 19$ & $0 / 0$ & $3 / 12$ \\
\hline Meses de seguimiento postrasplante & & & 1 \\
\hline Mediana (RIC) & $22(13-65)$ & $28(20-95)$ & $6(11-55)$ \\
\hline Rango & $1,7-105,7$ & $1,7-105,7$ & $2,4-88$ \\
\hline \multicolumn{4}{|l|}{ Meses entre diagnóstico SFM y TPH } \\
\hline Mediana (RIC) & $15(5-21)$ & $5(1-8)$ & $18(7-25)$ \\
\hline Rango & $1-46$ & $1-34$ & 446 \\
\hline $\mathrm{EICH}$ agudo, $\mathrm{n}(\%)$ & $7(29)$ & $3 / 10$ & $4 / 7$ \\
\hline EICH crónico, n (\%) & $7(29)$ & $2 / 6$ & $5 / 11$ \\
\hline
\end{tabular}

SFM: Síndrome de falla medular, SFMA: Síndrome de falla medular adquirida, SFMC: Síndrome de falla medular congénita, DE: desviación estándar, EICH: enfermedad injerto contra huésped, TPH: Trasplante de progenitores hematopoyéticos, RIC: rango intercuartil. 
Falla medular - D. Medina V. et al

Tabla 2. Casos de pacientes pediátricos con SFM en un centro de alta complejidad

\begin{tabular}{|c|c|c|c|c|c|c|c|}
\hline ID & $\mathrm{TPH}$ & Edad/género & Etiología de SFM & $\begin{array}{l}\text { Infecciones graves y/o } \\
\text { recurrentes al momento } \\
\text { de diagnóstico }\end{array}$ & ATG & $\begin{array}{c}\text { EICH } \\
\text { crónico }\end{array}$ & Resultado (vivo/muerto) \\
\hline 1 & No & $M / 15$ & Adquirida & No & Sí & NA & Muerto-infección \\
\hline 2 & No & $F / 9$ & Adquirida & Sí & Sí & NA & Muerto-infección \\
\hline 3 & No & $\mathrm{F} / 7$ & Adquirida & No & Sí & NA & Vivo \\
\hline 4 & Sí & $\mathrm{F} / 12$ & Adquirida & Sí & Sí & sí & Vivo \\
\hline 5 & Sí & $\mathrm{M} / 6$ & Adquirida & No & Sí & Sí & Vivo \\
\hline 6 & Sí & $F / 9$ & Adquirida & Sí & Sí & No & Muerto-adenovirus \\
\hline 7 & Sí & $M / 11$ & Adquirida & Sí & Sí & No & Vivo \\
\hline 8 & Sí & $M / 2$ & Adquirida & Sí & Sí & No & Vivo \\
\hline 9 & Sí & $M / 1$ & Adquirida & No & Sí & No & Vivo \\
\hline 10 & Sí & $\mathrm{M} / 6$ & Adquirida & No & No & No & Vivo \\
\hline 11 & Sí & $\mathrm{F} / 6$ & Anemia de Fanconi & No & No & No & Vivo \\
\hline 12 & Sí & $F / 12$ & Anemia de Fanconi & No & No & No & Vivo \\
\hline 13 & Sí & $\mathrm{M} / 7$ & Anemia de Fanconi & Sí & No & No & Vivo \\
\hline 14 & Sí & $\mathrm{M} / 9$ & Anemia de Fanconi & No & No & No & Vivo \\
\hline 15 & Sí & $\mathrm{M} / 5$ & Anemia de Fanconi & No & No & Sí & Vivo \\
\hline 16 & Sí & $F / 9$ & Anemia de Fanconi & Sí & No & Sí & Vivo \\
\hline 17 & Sí & $F / 12$ & Anemia de Fanconi & No & No & No & Muerto-adenovirus \\
\hline 18 & Sí & $F / 1$ & Disqueratosis congénita & No & No & Sí & Vivo \\
\hline 19 & Sí & $F / 1$ & Disqueratosis congénita & No & No & Sí & Muerto-progresión pulmonar \\
\hline 20 & Sí & $F / 3$ & $\begin{array}{c}\text { Trombocitopenia } \\
\text { amegacariocítica congénita }\end{array}$ & Sí & No & No & Vivo \\
\hline 21 & Si & $\mathrm{M} / 1$ & $\begin{array}{c}\text { Trombocitopenia } \\
\text { amegacariocítica congénita }\end{array}$ & Sí & No & No & Muerto-CMV \\
\hline 22 & Si & $\mathrm{M} / 2$ & $\begin{array}{l}\text { Anemia de Diamond } \\
\text { Blackfan }\end{array}$ & No & No & Sí & Vivo \\
\hline 23 & No & $M / 5$ & Anemia de Fanconi & No & No & NA & Vivo-soporte transfusional \\
\hline 24 & No & F/9 & Anemia de Fanconi & No & No & NA & Vivo-soporte transfusional \\
\hline
\end{tabular}

TPH: Trasplante de progenitores hematopoyéticos; SFM: Síndrome de falla medular; ATG: Globulina antitimocítica; EICH: Enfermedad injerto contra huésped.

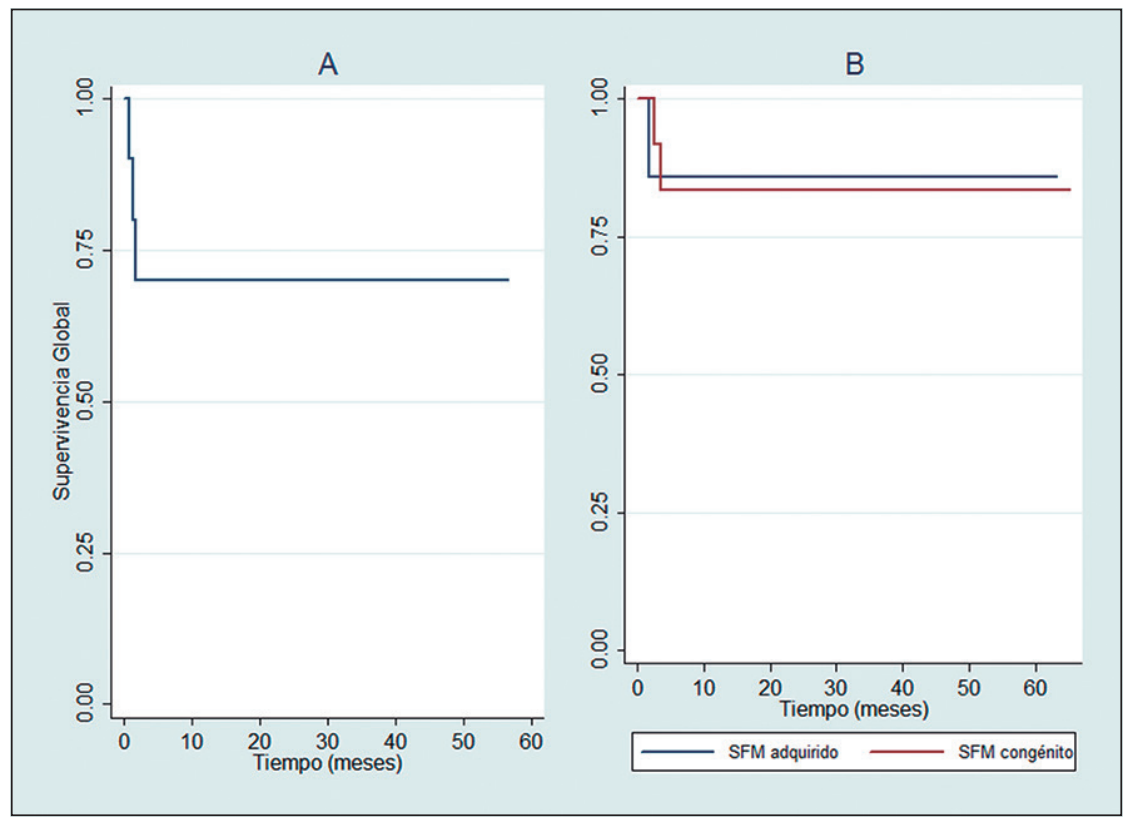

Figura 2. Supervivencia global del paciente con trasplante de progenitores hematopoyéticos (TPH) y diagnóstico de síndrome de falla medular (SFM). A) Pacientes con TPH y SFM. B) Pacientes con TPH según el tipo de SFM. 


\section{Discusión}

Se presenta una serie de pacientes pediátricos con SFM tratados en una institución de alta complejidad durante los años 2011-2017. Nuestra experiencia mostró que la etiología predominante fue el SFMC, lo cual difiere de los reportes en la literatura ${ }^{2}$ y podría ser explicada por el sistema nacional de referenciación de pacientes que incluye a esta institución como un centro de referencia para TPH. El diagnóstico congénito más frecuente fue AF, así como lo reportan en otras series ${ }^{21}$. En esta serie, para el grupo de SMFC la mayoría recibió $\mathrm{TPH}$, con una sobrevida a 5 años de $83 \%$. En el grupo de patologías adquiridas una gran proporción recibió TIS con ATG como primera línea de tratamiento. A pesar de esto, la mayoría requirió TPH como segunda línea de tratamiento, mostrando un comportamiento diferente a los resultados terapéuticos de países desarrollados con tasa de éxito en primera línea de tratamiento alrededor de $60 \%{ }^{22}$ (95\% CI: 65.3,76.6 y similar a países en desarrollo con $34 \%{ }^{23,24}$. En Colombia, un estudio retrospectivo en pacientes entre 4 y 60 años con SFM, que recibieron trasplante alogénico familiar idéntico entre los años 1993-2011, encontró que la mayoría de los casos (74,3\%) tuvo SFMA, 22,9\% AF y $2,9 \% \mathrm{ADB}$, pero sin especificar la proporción de menores de 18 años ${ }^{25}$.

Las pautas internacionales actuales sugieren el uso de la TIS, ATG y la ciclosporina como terapia de primera línea para pacientes con anemia aplásica severa que carecen de un donante HLA relacionado idénti$\operatorname{co}^{6,7,26}$. En nuestra serie, 9/10 fueron manejados con ATG como primera línea de tratamiento, de los cuales 6 pacientes requirieron tratamiento de segunda línea con TPH, que se pudiera interpretar como no respuesta al tratamiento de primera línea. Lo anterior, podría estar explicado de alguna manera por el uso de ATG de conejo, que es la que se ha utilizado en la mayor parte de los casos en nuestro centro, con la cual algunos autores han reportado una tasa inferior con respecto a la ATG de caballo, que podría asociarse a mejores resultados en pacientes con $\mathrm{SFM}^{27}$. Las tasas de respuesta hematológica varían, al menos en parte debido a la falta de consenso sobre los parámetros involucrados en su definición (independencia de la transfusión, mejora absoluta o relativa en los recuentos sanguíneos). Aproximadamente el $60 \%$ de los pacientes responden a los 3 o 6 meses después del inicio de $\mathrm{ATG}^{28,29}$. En nuestra serie, el tiempo entre el diagnóstico de SFMA y trasplante estuvo en la mayoría por debajo de los 8 meses, lo que sugiere tiempos cortos de no respuesta al tratamiento de primera línea.

El TPH es el tratamiento de elección para pacientes diagnosticados con falla medular moderada o severa ${ }^{1}$, idealmente de donante relacionado HLA idéntico ${ }^{30,31}$, prefiriendo como fuente la médula ósea, la cual se asocia a tasas menores de EICH. Sin embargo, también pueden emplearse de sangre periférica o proveniente de sangre de cordón umbilical ${ }^{32}$. Estudios recientes muestran buenos resultados utilizando trasplantes haploidénticos como primera línea o de rescate, incluso resultados comparables con donante relacionado idéntico, lo cual los convierte en una alternativa segura ${ }^{33-35}$. En esta serie se reportan igual número de TPH con donante idéntico y haploidéntico, con una SG del 84\%, lo cual coincide con reportes recientes en donde las tasas de SG son similares con diferentes tipos de donante ${ }^{13,26,33,34,36}$. En esta serie se observó EICH agudo y crónico en el $29 \%$ de los pacientes, similar a lo reportado en la literatura con diferentes tipos de donante, que están entre 21,1$47,7 \%{ }^{26,34,37}$. El estudio realizado por Sallfors-Holmqvist et al (BMTSS-2), entre 1974 y 2010, en el cual incluyeron pacientes menores de 21 años llevados a TPH debido a SFM, obtuvieron una SG de $86,4 \%$ a los 15 años posterior al $\mathrm{TPH}^{38}$. En nuestro caso alcanzamos una SG del $84 \%$ a 5 años, y específicamente $86 \%$ en el grupo de SFMA. Sin embargo, este procedimiento implica un gran riesgo de mortalidad relacionado y puede estar asociado con morbilidad temprana o tardía significativa ${ }^{6}$. Los casos de mortalidad relacionados con el trasplante en nuestra serie fueron 3 , que representaron un $15 \%$ del total de trasplantados, inferior al valor reportado por Muñoz-Villa, et al, quienes reportaron seis muertes en una serie de 27 pacientes $(22 \%)$ con $\mathrm{SFM}^{24}$.

Dentro de los factores de buen pronóstico identificados para pacientes que serán llevados a TPH están: ser menor edad, menor intervalo entre el diagnóstico y el trasplante, menor número de transfusiones previas al trasplante, uso de hemoderivados irradiados, menor número de infecciones pre-trasplante, mayor grado de histocompatibilidad donante-receptor, condicionamiento sin irradiación corporal total (ICT) y número suficiente de células progenitoras infundidas ${ }^{6}$.

En estos pacientes la principal causa de mortalidad relacionada al trasplante fue la complicación infecciosa, dentro de esta se encontró adenovirus y citomegalovirus como las más frecuentes. En la literatura se han encontrado resultados similares para donante relacionado y no relacionado, donde se mencionan las infecciones y las fallas de injerto como principales causas de muerte ${ }^{37,39-41}$, dentro de las infeccionas predomina la etiología fúngica seguida de la viral y posteriormente la bacteriana ${ }^{42}$.

Algunas limitaciones de este trabajo incluyen la recolección retrospectiva de la información que limitó el acceso a información disponible en las historias clínicas. Consideramos que este estudio podría tener un sesgo de referencia de pacientes para el tratamiento, 
dado que la institución es un centro de alta complejidad y referente nacional para remisión de pacientes de diversos lugares de Colombia que requieren TPH. Otra limitación fue la falta de información genética de los casos para discriminar de manera más precisa y completa los casos descritos.

En conclusión, los desenlaces observados en esta serie de casos de población pediátrica con SFM muestran similitudes con otras series en los resultados de SG para los pacientes trasplantados. La distribución de las patologías dentro de los SFMC, fue similar a lo reportado en la literatura. En esta serie fue mayor el número de casos con SFMC. En los pacientes con SFMA el número de pacientes que fueron tratados con TPH puede sugerir falla en el uso de ATG y con una supervivencia postrasplante favorable que permitió buenos resultados en ese grupo. En general la causa de muerte más frecuente fue de origen infeccioso, lo que también se ha reportado previamente en la literatura. El tratamiento instaurado en los pacientes de este estudio mostró resultados favorables en un centro de alta complejidad en un país latinoamericano.

\section{Responsabilidades Éticas}

Protección de personas y animales: Los autores declaran que los procedimientos seguidos se conformaron a las normas éticas del comité de experimentación humana responsable y de acuerdo con la Asociación Médica Mundial y la Declaración de Helsinki.

Confidencialidad de los datos: Los autores declaran que han seguido los protocolos de su centro de trabajo sobre la publicación de datos de pacientes.

\section{Derecho a la privacidad y consentimiento informa-} do: Los autores han obtenido el consentimiento informado de los pacientes y/o sujetos referidos en el artículo. Este documento obra en poder del autor de correspondencia.

\section{Conflicto de intereses}

Los autores declaran no tener conflicto de intereses.

\section{Referencias}

1. Dietz AC, Lucchini G, Samarasinghe S, Pulsipher MA. Evolving hematopoietic stem cell transplantation strategies in severe aplastic anemia. Curr Opin Pediatr. 2016;28(1):3-11.

2. Dokal I, Vulliamy T. Inherited aplastic anaemias/bone marrow failure syndromes. Blood Rev. 2008;22(3):14153.

3. Chirnomas SD, Kupfer GM. The inherited bone marrow failure syndromes. Pediatr Clin North Am [Internet]. 2013;60(6):1291-310. Available from: http://dx.doi.org/10.1016/j. pcl.2013.09.007.

4. Young NS, Kaufman DW. The epidemiology of acquired aplastic anemia. Haematologica. 2008;93(4):489-92.

5. Schoettler ML, Nathan DG. The Pathophysiology of Acquired Aplastic Anemia: Current Concepts Revisited. Hematol Oncol Clin North Am. 2018;32(4):581-94.

6. Milovic G, Ramos V, Rossi A, de los Milagros Touliet B. Síndromes de fallo medular. Soc Argentina Hematol [Internet]. 2017;300-4. Available from: http://sah.org.ar/docs/363-394.8.SAH GUIA2012_FalloMedular.pdf.

7. Sociedad Española de Hematología y Hemoterapia, Sociedad Española de Hematología y Oncología Pediátricas. Guía para el diagnóstico y tratamiento de las insuficiencias medulares
[Internet]. 2019.66 p. Available from: https://www.sehh.es/images/stories/ recursos/2019/03/20/Guia_GETH_ Diagnostico_Tratamiento_Insuficiencias_ Medulares_vFINAL_OK_con_bandera. pdf

8. Kulasekararaj AG, Mufti GJ, Marsh JCW. Bone marrow failure: causes and complications. Med (United Kingdom) [Internet]. 2017;45(5):265-9. Available from: http://dx.doi.org/10.1016/j. mpmed.2017.02.007

9. Philip Lanzkowsky Jeffrey M. Lipton Jonathan D. Fish, editor. Lanzkowsky's Manual of Pediatric Hematology and Oncology. 6th ed. 2016;102-33 p.

10. Calado RT, Clé DV. Treatment of inherited bone marrow failure syndromes beyond transplantation. Hematology. 2017;2017(1):96-101.

11. George B, Mathews V, Viswabandya A, Kavitha ML, Srivastava A, Chandy M. Fludarabine and cyclophosphamide based reduced intensity conditioning (RIC) regimens reduce rejection and improve outcome in Indian patients undergoing allogeneic stem cell transplantation for severe aplastic anemia. Bone Marrow Transplant. 2007;40(1):138.

12. Resnick IB, Aker M, Shapira MY, et al. Allogeneic stem cell transplantation for severe acquired aplastic anaemia using a fludarabine-based preparative regimen. Br J Haematol. 2006;133(6):649-54.

13. DeZern AE, Zahurak M, Symons
H, Cooke K, Jones RJ, Brodsky RA. Alternative Donor Transplantation with High-Dose Post-Transplantation Cyclophosphamide for Refractory Severe Aplastic Anemia. Biol Blood Marrow Transplant [Internet]. 2017;23(3):498504. Available from: http://dx.doi. org/10.1016/j.bbmt.2016.12.628

14. Thakar MS, Bonfim C, Sandmaier BM, et al. Cyclophosphamide-based in vivo T-Cell depletion for HLA-haploidentical transplantation in Fanconi anemia. Pediatr Hematol Oncol. 2012;29(6):56878.

15. Sanz J, Picardi A, Hernández Boluda JC, et al. Impact of graft-versus-host disease prophylaxis on outcomes after myeloablative single-unit umbilical cord blood transplantation. Biol Blood Marrow Transplant [Internet]. 2013;19(9):138792. Available from: http://dx.doi. org/10.1016/j.bbmt.2013.07.004

16. Jacobsohn DA, Vogelsang GB. Acute graft versus host disease. Orphanet J Rare Dis. 2007;2(1):1-9.

17. Klein OR, Buddenbaum J, Tucker N, et al. Nonmyeloablative Haploidentical Bone Marrow Transplantation with PostTransplantation Cyclophosphamide for Pediatric and Young Adult Patients with High-Risk Hematologic Malignancies. Biol Blood Marrow Transplant [Internet]. 2017;23(2):325-32. Available from: http:// dx.doi.org/10.1016/j.bbmt.2016.11.016

18. Robinson TM, O’Donnell PV, Fuchs EJ, Luznik L. Haploidentical bone 
marrow and stem cell transplantation: Experience with post-transplantation cyclophosphamide. Semin Hematol [Internet]. 2016;53(2):90-7. Available from: http://dx.doi.org/10.1053/j. seminhematol.2016.01.005.

19. Luznik L, O’Donnell PV, Fuchs EJ. Post-transplantation cyclophosphamide for tolerance induction in HLAhaploidentical bone marrow transplantation. Semin Oncol [Internet]. 2012;39(6):683-93. Available from: http://dx.doi.org/10.1053/j. seminoncol.2012.09.005.

20. Vaht K, Göransson M, Carlson K, et al. Incidence and outcome of acquired aplastic anemia: Real-world data from patients diagnosed in Sweden from 20002011. Haematologica. 2017;102(10):168390.

21. Peffault De Latour R, Peters C, Gibson B, et al. Recommendations on hematopoietic stem cell transplantation for inherited bone marrow failure syndromes. Bone Marrow Transplant. 2015;50(9):1168-72.

22. Rogers ZR, Nakano TA, Olson TS, et al. Immunosuppressive therapy for pediatric aplastic anemia: A North American Pediatric Aplastic Anemia Consortium study. Haematologica. 2019;104(10):197483.

23. Garanito MP, Carneiro JDA, Filho VO, Scheinberg P. Outcome of children with severe acquired aplastic anemia treated with rabbit antithymocyte globulin and cyclosporine A. J Pediatr (Rio J) [Internet]. 2014;90(5):523-7. Available from: http://dx.doi.org/10.1016/j. jped.2014.02.004.

24. Muñoz Villa A, Ortega Aramburu JJ, Bureo Dacal E, et al. Trasplante alogénico de médula ósea en niños con aplasia medular grave adquirida. Resultados a largo plazo. An Esp Pediatr. 1999;50(1):29-32.

25. Abello V, Villamizar L, Pedraza E, et al. Trasplante alogénico de progenitores hematopoyéticos para síndromes de falla medular, experiencia de la Unidad de Trasplante de Médula Ósea de la Clínica de Marly. Rev Colomb Hematol y Oncol [Internet]. 2012;1(1):44-51. Available from: http://www.imbiomed.com.mx/1/1/ descarga.php?archivo=CoHo121-05.pdf.

26. Bacigalupo A, Giammarco S.
Haploidentical donor transplants for severe aplastic anemia. Semin Hematol [Internet]. 2019;56(3):190-3. Available from: https://doi.org/10.1053/j. seminhematol.2019.03.004.

27. Yang N, Chen J, Zhang H, et al. Horse versus rabbit antithymocyte globulin in immunosuppressive therapy of treatmentnaïve aplastic anemia: a systematic review and meta-analysis. Ann Hematol. 2017;96(12):2031-43

28. Tisdale JF, Maciejewski JP, Núñez O, Rosenfeld SJ, Young NS. Late complications following treatment for severe aplastic anemia (SAA) with high-dose cyclophosphamide (CY): Follow-up of a randomized trial. Blood. 2002;100(13):4668-70.

29. Tisdale JF, Dunn DE, Geller N, et al. High-dose cyclophosphamide in severe aplastic anaemia: A randomised trial. Lancet. 2000;356(9241):1554-9.

30. Gluckman E, Wagner JE. Hematopoietic stem cell transplantation in childhood inherited bone marrow failure syndrome. Bone Marrow Transplant. 2008;41(2):127-32.

31. Samarasinghe S, Steward C, Hiwarkar $\mathrm{P}$, et al. Excellent outcome of matched unrelated donor transplantation in paediatric aplastic anaemia following failure with immunosuppressive therapy: A United Kingdom multicentre retrospective experience. Br J Haematol. 2012;157(3):339-46.

32. Asociación Colombiana de Hematología y Oncología Pediátrica ACHOP. Protocolo De Tratamiento Aplasia Medular Adquirida En Pediatria AMAACHOP-2017. 2017;28.

33. Dang BN, De Oliveira S, Gray A, Bowles LV, Moore TB. Successful engraftment of haploidentical bone marrow with post-transplantation cyclophosphamide in patients with aplastic anemia. Pediatr Transplant. 2020;(December 2019):1-5.

34. Lu Y, Sun RJ, Zhao YL, et al. Unmanipulated Haploidentical Hematopoietic Stem Cell Transplantation Achieved Outcomes Comparable With Matched Unrelated Donor Transplantation in Young Acquired Severe Aplastic Anemia. Biol Blood Marrow Transplant [Internet]. 2018;24(9):1881-7. Available from: https://doi.org/10.1016/j. bbmt.2018.05.015.

35. Young NS, Calado RT, Scheinberg P. Review in translational hematology Current concepts in the pathophysiology and treatment of aplastic anemia. Bloodjournal. 2006;108(8):2509-19.

36. Georges GE, Doney K, Storb R. Severe aplastic anemia: allogeneic bone marrow transplantation as first-line treatment. Blood Adv. 2018;2(15):2020-8.

37. Fagioli F, Quarello P, Zecca M, et al. Haematopoietic stem cell transplantation for Diamond Blackfan anaemia: A report from the Italian Association of Paediatric Haematology and Oncology Registry. Br J Haematol. 2014;165(5):673-81.

38. Holmqvist AS, Chen Y, Wu J, et al. Late Mortality after Allogeneic Bone Marrow Transplantation in Childhood for Bone Marrow Failure Syndromes and Severe Aplastic Anemia. Biol Blood Marrow Transplant. 2019;25(4):749-55.

39. Eckrich MJ, Ahn K-W, Champlin $\mathrm{RE}$, et al. Effect of race on outcomes after allogeneic hematopoietic cell transplantation for severe aplastic anemia. Am J Hematol [Internet]. 2014;89(2):1259. Available from: http://doi.wiley. com/10.1002/ajh.23594.

40. Bacigalupo A, Socié G, Schrezenmeier $\mathrm{H}$, et al. Bone marrow versus peripheral blood as the stem cell source for sibling transplants in acquired aplastic anemia: Survival advantage for bone marrow in all age groups. Haematologica. 2012;97(8):1142-8.

41. Chu R, Brazauskas R, Kan F, et al. Comparison of Outcomes after Transplantation of G-CSF-Stimulated Bone Marrow Grafts versus Bone Marrow or Peripheral Blood Grafts from HLA-Matched Sibling Donors for Patients with Severe Aplastic Anemia. Biol Blood Marrow Transplant [Internet]. 2011;17(7):1018-24. Available from: https://linkinghub.elsevier.com/retrieve/ pii/S1083879110004799.

42. Maury S, Balère-Appert ML, Chir Z, et al. Unrelated stem cell transplantation for severe acquired aplastic anemia: Improved outcome in the era of high-resolution HLA matching between donor and recipient. Haematologica. 2007;92(5):58996. 